\title{
Besprechungen und Notizen - Practische Notiz.
}

Aus 1·'rof. Dr. Litten's Poliklinix für innere Hautkrankheiten berichtet Dr. W. Rirschlaff über das Yorkommen und die Bedeutung eigenartiger Tiguren erweiterter Havtvenen am untercn Teil des Thorax in der

Deutschen Med. Wocheiisclir. 1894. "No. 11. Abgeselien von den Fallen allgemeiner Stauung, bei denen es zugleich zur Erweiterung des ganzen nervösen Apparates kommt, lassen sich für die Aetiologie des sich in der Thoraxhaut ausbildenden Venennetzes zwei Gesichtspunkte finden. Es ergeben sich Zustände, bei denen einerseits durch Behinderung des Blutabflusses zum Herzen übermässige Füllung der Hautnerven eintritt, andererseits solche, bei den durch Verschluss gewisser nervöser Wege sic A collaterale Bahnen in der Haut ausbilden, bei denen vielleicht vorlier mit blossem Auge nvch un-sichtbare Venen, weil von grossen Blutmengen durchrlossen. sichtbar werden; selbstverständlich können diese beiden Erschemungen gleichzeitig vorkommen. Da die Venen der vorderen Brustwand durch mehrere grössere Gel'ässo mit der Vena cava superior cominunicieren, so kann der völlige oder teilweise Verschluss dieser Gefässe durch Compression oder Thrombose oder durch bei-des Venectasie bewirken. Ursache für die Compression sind primäre oder metastatische Neubildungen im Mediastinalraume oder die zuweilen erst viele Jahre nach vorangegangeneii Exsudaten auftretenden Schrumpfungen der Pleura und des Pericard, welche die grossen Gefässe fast vollständig zur Obderation bringen können und so zum Tode führen. Von grossem Interesse sind sehliess-lich die innerhalb weniger Stunden sich ausbildenden Venenerweiterungen, die bei Thrombose der V. jugulares, subclaviae oder anonymae auftreten können.

Ausser den oben angeführten, durch grt.be mechanische Vorgänge in der Brusthöhle bedingten Erweiterungen grösserer venöser Gefässe kommen noch häufig zierliche Figuren von einer Reihe sich baumartig verästelnder Venen-stämmchen in der Nähe des unteren B,ippenrandes vor, von den Autoren, welche verschieden gedeutet sind, ohne dass bisher eine befriedigende Erklärung dafür ge-geben wurde. Eine solche glaubt Verf. numnehr gefunden zu baben. Die Intercostalvenen, die ein stets offenes Lumen zeigen, wirken bei der Rippenbewegung als Pumpe zum Vorteil des Blutlaufes; es besteht für ilen Abtiuss des Venenblutes am Thorax ein ähnlicher Saug- und Druckapparat, wie er für die oberen und unteren Extremitäten nachgewiesen ist. Dass nun die Venenerweiterungen nur am unteren Teil des Thorax vorkommen, findet seine anatomische Erklärung darin. dass die Mündungen der unteren Intercostalvenen in die V. azygos und hemiazygos nie oder nur ganz ausnahmsweise Klappeu besitzen, während sie bei den oberen selten fehlen. Ausserdem befürdert am oberen Thoraxteil die reichliche Muskel-masse den Blutabfluss besonders, was am unteren Teil nicht in dem Masse ge-schehen kann. Für das häuíigere Vorkommen des $\Lambda$ Гenenkranzos bei Männern spricht auch die Verschiedenheit des Athmungstypus beim männliclien und weib-liclien Geschlecht. Die Venenausdehnungen werden also bei alien Zuständen sichtbar sein. bei denen eine nicht genügende Ausdehnung des Thorax bei der Athmung stattfindet, wo keine genügende Contraction der Intercostalmuskeln, keine genügende Spannung der Pleura - die Ursache für das Klaft'en der Intercostalvenen - für den Blutabfluss sorgt. Dies ist besonders beim Lungen-einphysom der Fall, wo die inspiratorische 
Ausdehnung des Thorax nur gering ist. Mit dieser Affection waren ungefähr vier Fünftel dor Patienten, die den

$-480-$

Venenkranz zeigten, behaftet. Bei den übrigen, die zumeist eine geringe Aus-dehnungsfähigkeit der Lungen besassen, handelte es sich gewöhnlich um chronische Lungen- and Herzleiden. Verf. resumiert sich dahin, dass dem Venenkranz in der Nähe des unteren Rippenrandes cine diagnostische Bedeutung zukommt. ja, dass dem Phänomen eine prognostische Wichtigkeit in gewissen Fallen zu vindicieren ist, da durch dasselbe die Aufmerksamkeit auf Organerkrankungen liingelenkt wird, die später zu schwerwiegenden Erscheinungen í'ühren können. Edmund Saalfeld, Berlin.

Kanter. Ueber das Yorkommen von eosinophilen Zellen im malignen Lyiophoin und bei einigen anderen Lymphdrüsenerkrankungen. (Centralbl. f. Allg. Path. u. Path. Anat. 1894.) Kanter hat auf Jadassohn's Veranlassung die Gl·oldmann-schen Befunde nachuntersucht, die ein massenhaftes Auftreten von eosinophilen Zellen, zahlreicher Mitosen und eigentümlicher, extra und intracellulär gel $\theta$ gener kugelartiger Gebilde in malignen Lymphomen betrafen. Kanter's Material eutstammt den malignen Lymphomen eines dreijährigen Patienten, dessen Blut eine mässige Leukocytose, aber keine Vermeh $\pi \mathrm{mg}$ der eosinophilen Zellen zeigte. Die in S ·.iblimat Müller's < iher und Flemming'scher Flüssigkeit fixierten Schnitte wurden zum grössten Teil mit Ehrlich's Triacidlösung gefärbt und zeigten die ,weiehe Form” der malignen Lymphome. Eos $1 / 8$ ophíle Zellen fanden sich sehr reichlich, d. h. in jedem beliebigen Gesichtsfeld war eine grössere Zahl derselbon sichtbar; sie enthielton ein en, zuweilen gelappten, oder zwei Kerne, die von den Granu-lationen allseitig umschlossen werden und waren bedeutend kleiner, als die im normalen Knochenmark vorkommenden acidophilen Zellen. Kugelzellen, welche Goldmann ausschliesslich im Bindegewebsstroma í'and und als ein rötliches Proto-plasma beschreibt, das in einzelne regelmässige Felder zerfiel, welche sehr ver-schieden grosse Kugeln enthielten, konnte Kanter nur in einera Schnitte nach-weisen. Dieso Kugeln, die sich namentlich in Tumoren zu finden scheinen, will Goldmann, der sie ausser durcli die Bíondi’sche Färbung auch durch die Saffranintinction sichtbar machen konnte, mit den Flemming'tichen tingiblen Körporn in Zusammenhang bringen, oder als Haidenhain'sche Phagocyten auf-fassen, während sie Tanton mit den sog. lîussel'schen Fuchsinkörperchen identi-ficiert, welcher Ansicht sich auch Kanter anschliesst.

Mitosen fanden sich in sehr grosser Zahl und in den verschiedensten Stadien, ohno 'eine differentialdiagnostische Bedeutung zwischen Sarkom und malignem Lymphom zu haben, da bei beiden Erkrankungen das schnelle Wachs-tum der Tumoren cine reiehlichere Zellproliferation voraussetze. Dagegen ist Kanter der Frage, ob die eosinophilen Zellen eine diagnostische Bedeutung für das maligne Lymphom haben, näher ger,reten und hat zu diesem Zweck eine Anzahl von normalen und pathologischen Lymphdriisen auf diesen Punkt unter-sucht. Wiihrend die meisten Präparate einen negativen oder nur sehr spärlichen und schwankenden Befund an eosinophilen Zellen ergaben, fanden sich dieselbeu massenhaft in einer im Verlaufe einer hochgradigen Prurigo aufgetretenen. Inguinal-driise. Interessant war ferner, dass sich in einzelnen Fallen, so besonders in einer Peritonäaldrüse bei parametritischem Abscess, die Drüsenkapsel reichlich mit ihnen infillrierfc zeigte, wiihrend die Drüsensubstanz selbst beinahe frei von ihnen war, und dass sich die Zellen nur selten in den Gefässen, resp. in der Umgebung derselben fanden, auch wenn die Ge $\Lambda$ vebe reichlicher mit ihnen durchsetzt waren.

Aus diesem Grunde will Kanter die Goldmann'sche Ansicht, class die eosinophilen Zellen aus der Blutbahn in das Gewebe übertreten, nicht ohne Weiteres acceptieren. Er geht dabei von der 
V'oraussetzung aus, dass sich die Zahl der eosinophilen Zellen im strömenden Blute sowohl bei Prurigo als auch bei malignem Lymphom in den normalen Grenzen halte. (Für Prurigo trifft diese Voraussetzuug nicht zu, Ref. hat bei Prurigo die Zahl der eosinophilen Zellen stets, meistens recht bedeutend vermehrt gefunden.) Walâemar Peter.

Im British Journal of Dermatology No. 01, Vol. 5 berichtet J. F. Payne über zwei Fälle von XantL·oma deren Träger sich in Constitution, Gest'alt, Aussehen und Hauterscheinungen vollkommen glichen und sich nur dadurch

$-481-$

unterschieden, dass bei dem einen Zucker im Urin gefun den wurde, beim andern keiner vorhanden war. Von diesem letzteren wird der Befund mitgeteilt: Bräunliche, dunkelrosa bis hellgelbe Papeln oder flache Knötchen von Stecknadel-knopfgrösse, z. T. confluiert und dann bis linseng -ross, die sich hart anfühlten, bei Druck eine gclbere Nüancierung annahmen und leicht juckten, waren über die Ingaiinal- und Lumbargegend, an den unteren Extremitäten bis fast an die Kniee, auf den Scapulis und den Armen bis fast an die Handgelenke herab ver-streut. Die Affection war seit $1 \mathrm{Jahr}$ in 3 Sehiiben aufgetreten;. die Efñores-cenzen bestanden jedesmal einige Wochen bis Monate und gingen dann spontan mit Hinterlassung einiger braunpigmentierter $\Gamma$ lecke zuriick. Die augenblicklich vorhandenen Eruptionen waren vor 5 Wochen aufgetreten und schon in Rückbildung begriffen. In Anschluss an eine Casuistik über 13 analoge Fälle bei Diabetikern von Malcolm Morris und auf Grund der vom gewöhnlichen Hauthom differenten Ver-teilung, des Contiuierens, der Consistenz, des raschen Auftretens und spontanen Verschwindens, der leichten subjectiven Erscheinungen, des Fehlens von Leberaffectionen, der Constitution des Patienten und der bei Morris auch oft nur transitorischen Grlycosurie stellt Payne die Diagnose auf Xaathoma diabeticorum, trotz des Fehlens von Zucker im Urin. Únter indifferenter Therapie ver-schwanden die Erscheinungen ganz.

Die mikroskopische Untersuchu $\pi$, die an Schnitten aus einem dem diabetischen Patienten excidierten Hautstück gemacht werden musste, ergab: Leichte, zellige Infiltration im Corium, bosonders in der Umgebung der Drüsen; Vermehrung und Vergrösserung der fixen Bindegewebszellen, sowie teilweise der elastischen Fasern, Vorhandensein grosser (Xanthom) Zellen, z. T. granuliert, z. T. fettig degeneiiert. Diese Veränderungen sind partiell bei den verschiedenen Xanthomarten beschrieben worden; dass sie hier sämtlich bei einem Falle vereinigt sind, lässt den Verf. an wenigstens ein gemeinschaftliches ätiologisches Moment fur die einzelnen Formen denken oder sie als correspondierend mit den verschiedenen Entwickelungsphasen des pathologischen Prozesses erklären. Da schliesslich fast alle Patienten mit Xanthoma diabeticorum sich in einem überuormalen Ernährungszustande befanden und ihre Glycosurie nahezu immer vorübergehend war, schlägt er vor, die Affection als Xauthoma glycosuricum zu bezeichnen. Or. Born -Wildungen.

Dr. Eãuard Welander, Stockholm (Nordiskt Mediciniskt Arkiv. 1894. No. 13) veröffentlicht einen Fall von $\mathrm{G}^{1} / 8$ onorrlioe, mit Endocarditis saint cutanen Manifestationen compliciert, die genaue Krankengeschichte eines Patienten, $\Lambda$ velcher zweimal an Gonorrhoe erkrankte, jedesmal nach 1-2 Wochen heftige Ficbererscheinungen darbot, durch rheumatoide Schmerzen sowie einen Aus-schlag von hämorrhagisch - erythematösem Charakter compliciert, indem bei der zweiten Erkrankung sich noch Endocarditis sowie eine Affection der Xieren zugesellte. Der Ausschlag selbst bestand aus kleineren und grösseren, mehr oder weniger über die Oberfiäche erhabenen, teilweise mit Bläschen und Pusteln bosetzten Flecken, $\Lambda$ velche nicht juckten und an 
den Unter- und Ober-schenkeln, Armen und Rurapf auftraten. Der Inhalt der Blasen war bacterien-frei, in die Urethra eines Gesunden gebraeht, rief er keine Erscheinungen hervor, die Impfung auf den Arm des Verf. blieb ohne Folgen. W. glaubt den Ausschlag auf die Wirkung von Toxinen zurückführen zu müssen, während er es dahingestellt sein lässt, ob die Endocarditis direkt durch die Gonokokken oder erst mittelbar durch andere Bacterien, welchen die Gonorrhoe den $\Lambda$ Veg geebnet hatte, hervorgerufen wurde und möchte daher nur von einer paragonorrhoischen Affection sprechen, er will aber diesen Fall als eine Stütze der jetzt immer mehr vertretenen Ansicht verwertet wissen, dass die Gonorrhoe nicht einfach als locale Erkrankung angesehen werden darf, sondern eventuell auch den Charakter einer AllgeineinInfection annehmen kann.

Im Archiv für Dermatologie und Syphilis (XXVII, 2) ist ein Vortrag „Ueber die Häuíigkeit und Zeit des Auftretens der Urethritis posterior bei $<$ ler acuten Gonorrhoe" erschienen, den Alfred Lanz (Moskau) auf dem V. russi-schen Aerztekongress zu St. Petersburg gehalten hat. Zuerst $\Lambda$ vies der Vor-tragende auf die Ursache der verschiedenen Resultate der bisher über diese Dermatologisehe Zeitschrift, Band I. Heft 5. '

$-482$

Frage atigestellten Untersuchungen hin. Während die einen Autoren nur erstmalige Gonorrhoeorkrankungen in das Bereich ihror Untersuchungen gezogen, haben andere die Sonderung von erstmaliger und wiederholter Erkrankung nicht gemacht; hierin liege eine Fehlerquelle, weil oft eine Urethritis post, noch von der vorigen Infection zurückgeblieben sein kann. Ferner unterscheiden sich die bisherigen Veröfl'entlichungen dadurch, dass zur Beobach tung Fälle von vorschiedener Krankheitsdauer herangezogen wurden. Sodann unterzieht Vortragender die bisher angegebenen Mittel zur Diagnose der Ureth ritis post, einer kritischen Betrachtung und kommt zu dem Resultate, dass zwar die Lohnsteirische Methode von alien die genaueste ist, indes gestatte auch diese nicht, die Diagnose in alien Fallen auf Grand einer einmaligen Untersuchung zu stellen. Vortragender hat sich nun der Aufgabe unterzogen, in welchem Prozentsatz aller Fälle von acuter Gonorrhoe mit der Thompson'schen Zweigläserprobe eine Urethritis post, nachgewiesen werden kann, da er die Ueberzeugung hat, dass diese Probe in acuten Fallen mit reichlicher Secretion ganz zuverlässige Resultate ergebe. Unter 76 Fallen von acuter erstmaliger Gonorrhoe wies er in 61 Fallen $=80,3$ pOt. die Urethritis post, deutlich nach, so dass sein Resultat mit dem von Heisler gefundenen (79,71 pCt.) fast übereinstimmt. Er nimmt sogar an, dass in einigen der restierenden 15 Fälle das negative Resultat darauf beruhe, dass die Thompson'sche Probe bei einer Ureth ritis post., die sich schleichend und ohne reichliche Secretion ausbildete, unzuverlässig sei. Was die Zeit des Auftretens der Urethritis post, im Verlauf des acuten Trippers anbetriff't, so $\Lambda$ veicht sein Ergebnis von dem der andern Untersucher ausser Heisler bedeutond ab. Während jene in der grössten Zahl der Fälle die zweite oder dritte Woche angaben, bildete sich in seinen Fallen die Urethritis post, meistens im Laufe der ersten und zweiten Woche aus.

Diesen Termin konnte er deshalb meist genau feststellen, weil einerseits in der Mehrzahl der Fälle die Kranken vor dem Auftreten der hinteren Entzündung zu ihm kamen, andererseits er dieselben auf deren Hauptsymptome aufmerksam machte, und er sie beim Erscheinen derselben sofort zu sich bestellte. 
Er betrachtet diese Erkrankung nicht als eine Complication, sondern als ein ge.wisses Entwickelungsstadium der acuten Gonorrhoe, indem er zwar zugiebt, dass gewisse Momente das Auftreten der hinteren Entzündung beschleunigen könnten, aber nicht als bestimmte Ursachen anzusehen sind. Ferner kann Vortragender auf Grund seiner Erfahrung nicht der Ansicht beistimmen, dass die Einspritzungen ein schnelleres Uebergreifen der Entzündung auf die hintere Harnröhre bewirken sollten, im Gegenteil, er berechnet aus seinen Fallen, dass die Injectionen das Auftreten der Urethritis post, hinausschieben. Er erwähnt einen Fall, wo nach Einführung von Jodoformstäbchen die Urethritis post, schon nach 5 Tagen auftrat, wofür man wohl als Ursache den directen Transport des blenorrhagischen Eiters in dem hinteren Harnröhrenabschnitt ansehen muss. Der optimistischen Anschauung von A. Wolff, der bei frühzeitigem Beginn der Behandlung in der Mehrzahl der Fälle das Beschranken der Entzündung auf den vorderen Teil erreichen will, Iritt er entschieden entgegen. In einigen seltenen Fallen fand er, dass die Urethritis post, ohne jegliche subjective Symptome sich ausbildete; natürlich kann dann die rechtzeitige Diagnose nur durch continuierliche Untersuchung des Harns gestellt werden. Edmund Saalfeld-Berihi.

Giebt es eine idiopathische Nebenhoden- und Hodenentzündung i Von

Dr. A. Elsenberg. (Wiener med. Presse. 1893. No. 31 und 32.) Verf. wirft die Frage auf, ob ebenso wie in den Tropen auch in unserem Klima selb-ständige, idiopathische Hoden- und Nebenhodenentzündungen vorkommen können. Er führt drei Fälle eigener Beobachtung an, welche Analoga für die in den Tropen häufig gemachte gleíche Beobachtung bilden. In diesen Fallen handelt es sich utn Patienten, bei welchen plötzlich ohne erkennbare Ursache - Lues und Tuberculose konnten ausgeschlossen werden, Gonorrhoe hatte nie bestanden, Infectionskrankheiten, wie Parotitis, oder Malaria lagen nicht vor - Epididymitis und Orchitis mit roehr oder weniger heftigen Schmerzen auftraten und erst nach 2 bis 3 Wochen allmählich verschwanden. Verf. teilt dann zwei weitere, bis dahin einzig dastehende, Beobachtungen mit, deren eine sich auf einen Pat. bezieht, welcher wenige Tage vor Ausbruch des Masernexanthems eine äusserst schmerzhafte Epididymitis und Orchitis darbot, welche $-483-$

mit dem Beginn des Exanthems sich auffállig verminderte, während der andere Pat. wenige Tage nach einem Influenzaanfall ebenfalls an schmerzhafter Epi-didymitis und Orchitis erkrankte; bei beiden Patienten konnte Gonorrhoe absolut sicher ausgeschlossen werden. Während Verf. in den beiden letztgenannten Fallen die Ursacbe in der i-eitlich ziemlich congruenten lnfectionskrankbeit sucbt, glaubt er in den vorher citierten drei Fallen eine idiopathische, vielleicht durch ein bis jetzt noeh unbekunntes Virus hervorgerufene, Krankheitsform annehmen zu miissen.

Auf alle Fälle sind diese Mitteilungen, welchen Standpunkt man auch der Anschauimg des Verf. gegenüber einnelimen mag, interessant genug, urn auch andere Beobachtor zur $\Delta$ uftnerksamkeifc in entsprechenden Fallen anzuhalten. M. Friedlaen $<$ Ur--Be $\gamma \backslash$ hi.

Ueber Myxödem. Von Dr. S. J. Meltzer. (New-Yorker Medicinische Mvnatsschrifc April 1894.) Seitdem man nach den grundlegenden Versucben Schiffs angefangen hat, das Myxödem durch Darreichung von Tíerschilddriise zu bekämpfen, haben die Methoden der Einverleibung des Mittels in den Körper des Patienten schnell gewechselt. Die Implantation wurde verlassen, weil die Tierdrüse, auch wenn sio einheilte, ziemlich schnell resorbiert wurde; neuerdings ist auch die 
subcutane Injection von Schilddrüsensaft durch die Darreichung der Druse per os im allgemeinen verdrängt $\Lambda$ vorden. Die bequomste und brauch-barste Verabreichungsart scheint die Pulverform zu sein. Einen Fall, der mit diosor Behandlungsweise nahezu zur Heilung gebracht ist, stellte Meltzer im Herbst V. J. in dor deutschen Medizinischen Gesellschaft der Stadt New-York vor. Seine Patientin ist eine verheirathete Krau von 37 Jahren, die seit vielen Jahren in ärztlichor Behandlung war. Erblich ist sie nicht belastet. Als Verf. sie in Behandlung nahni, bot sie das typische Bild des Myxödems: Starkes, un-förmliches Aussehen, graugelbe Gesichtsfarbe; die Lippen sind stark cyandtisch. Augenspalte schmal, beide Augenlider stark verdiekt. Das ganze Gesicht scheint stark geschwollen, dvch hinterlässt der Fingerdruck keine Delle. Der gauze Körper ist mit ödematösen Geschwulstmasson bedeckt. Die Thyreoidea ist nicht zu íuhlen. Die Intelligenz ist langsam, aber gut; nur das Gedächtnis hat ge-litten. Das Aussehen ist sehr stupid, „cretínoid”. Sie kann kaum eine Thätig-keit im Haushalt richtig besorgen - der Zustand ist ein ganz unerträglicher.

Die Behandlung begann am 23. November 1893 und bestand in der Ver-abreichung von pulverisierter Schafsschilddrüse per os. Es wurde mit 5 Gramm täglich begonnen. Bereits nach einer Woche traten wahrnehmbare Veränderungen auf. Die Dosis wurde gradatim bis 15 Gramm erhöht , bei welcher Dosis im Januar d. Js. ein kritischer Anfall auftrat: Erbrechen, Appetitlosigkeit, Tem-peraturerhöhung und Pulsbeschleunigung. Nach Aussetzen des Mittels erholte sich die Patientin sehr schnell und es wurde ihr dann bis heute jeden vierten Tag 10 Gramm verabreicht.

Die Veränderung im Befinden und Aussehen ist heute eine gewaltige.

Sie hat an Gewieht 45 Pfd. verloren - das Anfangsgewicht betrug 225 Pfd. -

Gang und Sprache ist normal. Die Haut hängt stellenweise wie ein leerer Sack und fühlt sich geschmeidig an. Der Gesichtsausdruck ist total verändert, die psychischen Störunge ' sind bis auf einen geringen Grad von Reizbarkeit geschwunden; im übrigen ist die Patientin heiter und arbeitet fieissig. Am besten geben die photographischen Aufnahmen der Frau vor und während der Be handlung ein Bild der eingetretenen Besserung. Vollständige Heilung ist noch nicht da; doch hofft der Verfasser die Patientin in einigen Monaten völlig zu heilen, obschon sie ihr Lebenlang in jeder Woche 10-15 Gramm einer Thyre oidea wird nehmen miissen. H. Krey-Berlin.

Douglas \V. Montgomery: Atheroma. (The Medical News, February 17. 1894.) Der überraschend schnelle Heilungsverlauf der Wundhöhlen nach Athe-romenucleationen giebt dem Verf. Veranlassung, auf die pathologische Anatomie der Atheromkapsel ehvas genauer einzugehen. Das Atherom entsteht durch Einstülpung und Abschniirung eines Stückes normaler Haut, deren fortwährend abgestossenes Epithel, als Detritus in Kugelform angesammelt, eine hornartig glänzende Oberfläche erhält, welche mikroskopisch nur abgeplattete, kernhaltige $7^{*}$

$-481-$

Epithelien, aber keinerlei Bindegewebselemente zeigt. Sie bildet die Kapsel der Geschwulst, die gewöhnlich enucleiert wird. Die innere Bekleidung des nun be-stehenden Hohlraums ist aber erst die eigentlichc Atheromkapsel. Ihre Ober-fläohe entspricht deni Rete Malpiglii, unter ihr liegen die normalen Outisschichten normaler Haut. Um also eine Radicaloperation vorzunehmen, müsste man diese mit entfernen, während bei dem meist geübten Verfahren, das sich mit der Enucleation begnügt, nur durch den Hautsehnitt eine Wunde gesetzt wird, von deren mehr oder weniger aseptíschen Zustande die Heilung allein abhängt. 
Dr. .Borw-Wildungen.

In No. 15 der „Deutschen medizinischen Wochenschrift 1894” macht Max

Wakmen auf die grosse Resorptionsfähigkeit der Haut für Lösungen von

Arzneistoffen im Klever'schen Vasogen aufmerksam. Er selbst hat sich mit

D/gprozentigem Jodoformvasogen eingerieben und konnte bereits naeh 7 Stundon

Jod im Hani nachweisen, welches nach 22 Stunden verschwand. Ein gleiches

Resorptionsvermögen beobachtete Bayer bei Phthisikern, die mit Kreosotvasogen

behandelt wurden. Schon nach zweimaligem Einreiben fanden sich regelmässig

Phenole im Harn. Bei längerer Behandlung muss die Haut nach der Einreibung

sorgsam abgewaschen werden, da sie durch das Kreosot volltsändig pergamcnt-

artig und undurchlässig wird. In weitaus grösserem Masse wirken die Vasogene,

wenn sie auf Schleimhäute und Wunden appliciert werden. Sehr warm ompfiehlt

Hollstein das Jodoformvasogen für die Behandlung von Unterschenkelgeschwiiren

und Fisteln. Jirøy-Berlin.

In der Internationalen Klinischen Rnndschau (1894, No. 3) bespricht UUmann das Resultat der üntersuchungen, die liber die Verwendbarkeit des Europhens in der Dermatotherapie in der H.

von Hebra'sch $\beta$ n Poliklinik während zweier Jahre bei 176 Patienten mit gewissen venerischen und Haut-affectionen angestellt worden sind. Als günstige physikalische Eigenschaften erwähnt Verf. den hohen Jodgehalt (27,6 pCt.), die nahezu völlige Geruchlosig $\div$ keit, die dem Jodoform gegenüber günstigen Lösungsverhältnisse, das geringe specifische Gewicht (Europhen ist fünf mal so leicht als Jodoform), sowie die völlige Ungiftigkeit. Das Europhen kam zur Anwendung als Pulver, gelöst in Form des Aetherspray und als Salbe (mit Lanolin und Vaselin aa) und zeigte beim Ulcus molle dieselbe günstige Einwirkung wie das Jodoform, ohne desscn Nachteile zu besitzen. Auch bei ulcerierenden syphilitischen Primäraffecten oder nässenden Papeln an den Genitalien übte das Europhen stets eine reinigende und heilende Wirkung, analog der des

Jodoform, aus. Als sehr zweckraässig zur schliesslichen Uebernarbung der harten Geschwüre hat sich ein combiniertes Ver-fahren erwiesen. Die Geschwüre wurden Nachts mit grauem Pilaster bedeckt, und am Tage nach Entfernung des Secretes mittelst schwacher antiseptischer Lösungen mit Europhen eingestreut, wobei die Adhäsionsfähigkeit und die Gerucfilosigkeit des Europhens sich als sehr vorteilhaft erwiesen. Auch bei reinen, nicht inficierten Operationswunden hat sich das Europhen als Ersatz des Jodoforms erwiesen. Ausser bei den genannten Añ\%ctionen kam das Europhen erfolgreich zur Anwendung gegen locale , oberflächliche Geschwürsprozesse als õprocentiger Aethersyrup, ferner gegen Brandwunden als 3procentige Salbe (mit Vaselin und Lanolin aa).

Verf. resumiert sich folgendermassen: „Das Europhen hat sich uns demnach bei verschiedenen obertiächlichen Geschwürsprozessen, insbesondere aber den venerischen Geschwüren, sowie zum Verbinden reiner Wunden als bestes Ersatzmittel für das Jodoform bewährt. Keineswegs auf der gleich niedrigen Stufe mit so vielon in letzter Zeit als Jodoformersatz angepriesenen Mitteln stehend, wird sich das Europhen wegen seiner unleugbar kräftigen Wirkung und der schon genannten physikalischen Eigenschaften auch dauernd im Arzneisehatz erbalten." Saalfeld-Be $\Lambda \mathrm{m}$.

Im Archiv für Dermatologie und Syphilis (XXVH, 2) veröffentlicht Dr. K. E. Linden (Helsingfors) seine Untersuchungen, die er über die iíe-haudhing: der Syphilis mit Injectionen von Calomel und salicylsaurem Quecksilber (resp. Thymolquecksilber) angestellt hat. Verf. beruft sich darauf, 
$-485-$

dass das von ihm herangezogene Krankenmaterial, welches zum grössten Teil dem

Garnisonskrankenhause in Helsingfors entstammt, sick besser íiir die Unter-suchung, als solches aus biirgerlichen Krankenhäusern oignet, da es dio Forde-rung der inöglichsten Gleichmässigkeit in kokem Grade erfiillt. Es kandelt sich urn Patienten, die als zum Kriegsdienst tauglich, sonst von gesunder Körper-constitution sind, in ungefähr gleichem Alter, unter denselben hygienischen Ver-liältnissen sich befinden, und deren Behandlung und Beobachtung die gleich sorg-fältige ist. Lm ganzen hat Verf. 106 Fälle zum Vergleich herangezogen, unter diesen befanden sich 84 stationär im Krankenhause, 22 entstamraen jedoch der Privatpraxis, ihr Alter war dasselbe wie das der Brstgenannten. Die Injectionen, deren Dosis gewöhnlich 0,05 und 0,1 betrug, wurden stets subcutan in die Tro-chantergegend, mitunter zwischen den Schultern, in Zwischenräumen von V-/qi Wo-chen, vorgenommen. Was die Calomelinjectionen betrifft, so wurde das Calomel anfangsin Glycerin versohlemmt, später in Oel, weil in dieser Art die Häufigkeit der Abscesse abnahm, besonders seitdem das Oel gekocht wurde: auf möglichst strenge Antisepsis wurde selbstverständlich geachtet. Die Abscesse traten gewöhnlich nach Injectionen von grösseren Dosen auf. Ferner stellten sich auch zahlreiche, mehr oder weniger schmerzhafte bis faustgrosse Inflltrationen ein, deren Dauer oft mehrero Wochen betrug. Bei Injectionen von kleineren Dosen waren die localen B,eizer-scheinungen und Schmerzen geringer. Als Suspensionsmittel für Salicyl- und Thyruol-quecksilber verwandte Verf. Paraffinum liquidum, und zwar machte er von einer 10procentigen Mischung die Injectionen in Dosen von 0,1 und 0,05. Abscesse traten in keinem Fall auf, die in $5 \mathrm{pCt}$. aufgetretenen Inflltrationen vcrliefen alle indolent unter geringen Beschwerden, gewöhnlich traten sie am ersten Tage auf und verschwanden zumeist am zweiten oder dritten Tage. Binen Unter-schied zwischen dem Salicyl- und Thymolquecksilber hat Yerf. nicht gefunden. Infolge der leichten Lösbarkeit des Präparates traten sehr bald nach der Injection eine schnelle Resvrption andeutende Symptome auf, jedoch keine schweren Allgemeinsymptome, und leichte Stomatitis, keine einzige Enteritis. Der häuflg vermehrte Speichelfiuss, der sich gewöhnlich nach der 1. Injection am ersten Tage oft schon wenige Stunden nach der Injection zeigte, hörte meist am zweiten oder dritten Tag auf, analog dem Auftreten und Verschwinden des Quecksilbers im Harn. In 24 pCt. der Fälle trat nach der ersten Injection während des ersten oder zweiten Tages, wo der Organismus die grösste Quecksilbermenge aufnimmt und ausscheidet, eine auch noch von anderen Autoren beobachtete Temperatursteigerung auf: sie war gewöhnlich von Frost und Kopfschmerzen begleitet. In der überwiegenden Mehrzahl der Fälle trat die Steigerung nach der ersten Injection auf und konnte dann 3S,5 bis $40^{\circ} \mathrm{C}$. erreichen; mitunter wurde auch nach den späteren Injectionen Fieber beobachtet, indessen niedriger urn $38^{\circ}$ und von kürzerer Dauer. Verf. stellt das Fieber gleich dem, das durch solche Stoffe hervorgerufen wird wie Tuberculin. Was nun die Recidive anbe-trifft, so ist Verf. zu folgendem Resultat gekommen: Er empfiehlt die Excision der primären Sklerose in Fallen, wo es ohne grössere Ungelegenheiten geschehen kann, da in den Fallen, wo dies geschehen, das Recidiv entweder ganz aus-geblieben oder doch erst nach längerem Zwischenraum und dann mit leichtem Verlauf eintrat. Von alien Fallen blieben nach Injectionen von Calomel Recidive in 11 pCt. und nach Injectionen von Hydrarg. salicyl. und thymol, in 13,3 pCt. aus. Die Anzahl der Recidive war im allgemeinen nach Calomelinjectionen während des ersten Jahres geringer und die Intervalle zwischen den Recidiven länger als bei Anwendung der anderen Präparate, wogegen bei diesen die Recidive, wenn auch grosser an Zahl, in kürzerer Zeit verliefen; nach Injectionen von Hg. salicyl. trat eine geringere Anzahl von Spätrecidiven auf. Was die Zoitdauer der ersten Behandlung im Vergleich 
zu der der Recidive anbetrifft, so erforderte die erste Cur bei beiden Injectionsmethoden eine längere Zeit, als die der darauf folgenden Recidive. Während nun die Calomelinjectionen bei der ersten Behandlung schneller wirkten als die von Hg. salicyl. und thymol., so war die Dauer der Cur mit beiden Präparaten bei Recidiven ungefähr eine gleiche. Die Menge des Medikaments war bei den Recidiven etwas geringer als bei der ersten Kur.

Verf. resümiert sich dahin, dass das Calomel, trotzdem es ein stark wirkendes Präparat ist, welches in kürzerer Zeit die Symptome zum Schwinden bringt als Hg. salicyl. und thymol., doch diesen beiden nicht absolut vorzuziehen ist, da diese nicht

$-486-$

nur geringere locale Rcizung verursaclien, sondern auch den Verlauf der Kraiikheit überhaupt abzukürzen scheinen. indess stellt Verf. den Nutzen des Präparates nicht in Abrede bei Fallen, wo eine schnelle Wirkung erzielt werden soil.

Saalfeld-Bßriin.

H. Gillet, Arzt an der Pariser Kinderpoliklinik, berichtet in den Annalen der Pariser Polikliuik über 11 Fälle von teils hereditärer, teils erworbener Syphilis bei Kindern, welche mit dem Calomel pilaster des leider zu früh verstorbenen Quinquand behandelt sind. Dieses Pilaster besteht axis:

Em pi. diachyl 3000,0

Calomel vapore parat .... 1000,0

01. ricin . .300,0

mid wird in der Weise ausgestrichen, dass jeder Quadratdecimeter 1,20 Calomel enthält. Das Pilaster wird in Stücken von 10:15 cm auf den Körper geklebt, bleibt 8 Tage liegen, dann wird das Kind gebadet und ein neues Pilaster auf-geklebt, wozu man natürlich eine andere Stelle wählt.

Von den 11 Fallen scheidet einer gleich aus. da derselbe auch anders behandelt ist. Von den restierenden 10 Fallen sind nur 2, bei denen die Er-scheinungen geschwunden; die übrigon befanden sich teils noch in Behandlung, teils $\Lambda$ varen sie an intercurrenten Krankheiten gestorben, teils aus der Behandlung fortgeblieben. Wenn auch diese Erfolge nicht gerade sehr ermutigend sind, so verdient der Vorschlag immerhin der Beachtung, da er anwendbar ist, wenn irgend welche Umstände eine andere Behandlung verbieten.

F. Schütte-Bßñin.

Gíegen Lupus hilft nach Chotzens Erfahrungen (D. med. Wchschr. No. 46, 1893) das Alumnol in Form 1-2 procentiger Umschläge und gleichzeitiger Injection von 1 procentiger Lösung, subcutan in die Randzonen appliciert. Günstige Ergebnisse, welche der Autor in der medicinischen Section der Schles. Ges. f. V. 0. in Breslau demonstriert hat, ermutigen zur Nachprüfung dieses Verfahrens.

L.

Practische Notiz.

Die Ulcerationen in Raclien- und Mundschleimhaut, welche im Gefolge

von Mercurialcuren auftreten, kommen bekanntlich raanchmal erst nach Abschluss derselben, selbst wochenlang später zum Vorschein. Vielfach werden sie dann fiir Recidive der Krankheit selbst gehalten, von neuem mit speciflscher Allgemeinbehandlung bedacht und dadurch zuweilen in einen erschreckenden Grad der Verschlimmerung gebracht. Meist ist der Geschwtirsboden dann so gelockert und gereizt, der eiterige Schleimbelag so zäh und dickpelzig, dass die gewöhnlichen Aetzmittel, namentlich Lapis, aber auch die sonst so zuverlässig wirkende Chroni- 
säure im Stich lassen, ja auf die Touchierung mit diesen Präparaten sogar ein erneuter Reiz-Zustand í'olgen kann. Die Ulceration , durch jede Schluckbewegung gezerrt, kommt dann selbst bei Fortlassung aller Hg.-Präparate nicht zur spontanen Ausheilung. Wird nun irrtümlicher Weise aufs neue wiederum zum Mercur gegriti'en, so koinmen jene gangränösen Phlegmonen zu Stande, die auf die Choanen übergreifen und eine kaum heilbare Ozaena oder Kiefernekrose herbeizuführen piiegeii. Zuständen jener Art gegeniiber empfiehlt sich deshalb von vornherein ein thunlichst schonendes und doch energisch wirkendes Localmittel und als solches muss nach meinen Erfahrungen das Pyoctaninum caeruleum bezeichnet werden. Mit der concentrierten Methylenblau-Lösung tupft man den Geschwürsboden täglich ein oder mehrere Male reichlich ein, bis er sich ganz vollsaugt und lässt dann - wenn man die mit dem Ausspiilen sonst unvermeidliche Blaufärbung der Lippen vermeiden will - reichlich Wasser nachtrinken. Das Pyoctanin bewährt sich zu dem Zweck der Geschwürsreinigung vortrefflich und besitzt neben seiner antifermentativen Energie und Unschädiichkeit auch den Vorzug, dass die Application gegeniiber den Aetzmitteln weder Gewebsreaction noch den geringsten Schmerz hervorruft. Lassar. 